\title{
Mucopolysaccharidosis Type IIIC
}

National Cancer Institute

\section{Source}

National Cancer Institute. Mucopolysaccharidosis Type IIIC. NCI Thesaurus. Code C84899.

A rare autosomal recessive lysosomal storage disease caused by deficiency of the enzyme acetyl-CoA:alpha-glucosaminide acetyltransferase. It is characterized by behavioral changes, sleep disturbances, and mental developmental delays. 